\title{
Highly polarized burst of a water maser in Orion-KL
}

\author{
Shinji Horiuchi \\ National Astronomical Observatory, Mitaka, Tokyo, Japan \\ Osamu Kameya \\ National Astronomical Observatory, Mizusawa, Iwate, Japan
}

\begin{abstract}
It has been reported that the $22 \mathrm{GHz}$ water maser in the star forming region Orion-KL has started an outburst in rate 1997 (Omodaka et al. 1998, IAUC 6893). Using Mizusawa radio telescope we started a monitoring observation of the bursting maser. We measured the linear polarization of the maser after the burst, during a phase of rapid flux density decrease. We find that the total flux density of $2.4 \times 10^{6} \mathrm{Jy}$ (December 1998) exhibits about $46 \%$ linear polarization. Over the next six months we find that the total intensity decrease about two orders of magnitude while the fractional linear polarization gradually fell to $30 \%$. These results suggest that the present bursting phenomenon has an origin similar to the super maser event starting in 1979, and the phenomenon of the extremely bright masers in this region is geometric in nature and related to the strong magnetic field.
\end{abstract}

\section{Introduction}

The star-forming region Orion-KL is known to have once had a spectacular water-maser burst event accompanied by very high fractional polarization up to $70 \%$ in the 1980's (eg. Garay et al. 1989, Abraham, Vial Boas 1994). In December 1997, it was discovered that the Orion-KL water maser had again started to burst (Omodaka et al. 1998). The total flux density of the spectral feature at a velocity of $V_{L S R} \simeq 7.9 \mathrm{~km} / \mathrm{s}$ appeared to increase exponentially and reached the values as large as $\sim 3.5 \times 10^{6} \mathrm{Jy}$ in September 1998. The position of the present burst coincides with that of the previous event to an accuracy of 1" (Omodaka et al. 1999). As the present event is the only second known in this region, we can not judge periodicity.

The polarization properties of these two events might illuminate some aspects of the nature of the bursts. In this paper we report the result of polarization observations with a radio telescope during the period when bursting flux is rapidly decreasing. 


\section{Single dish monitoring}

On 1998 December 21 we started measurments of the linear polarization of the maser using the Mizusawa $10 \mathrm{~m}$ radio telescope. Soon we realized that it was during a phase of rapid flux density decrease after the burst. The detail result observations until June 8 can be seen in Horiuchi \& Kameya 2000. We continued the monitoring and the results up to February 2001 is shown in Figure 1a. The variations of total flux of the burst feature is shown in Figure 1b for the previous event in 80's (top, adopted from Garay et al. 1989), and for the present event (bottom, Omodaka et al. 1998, and this work).
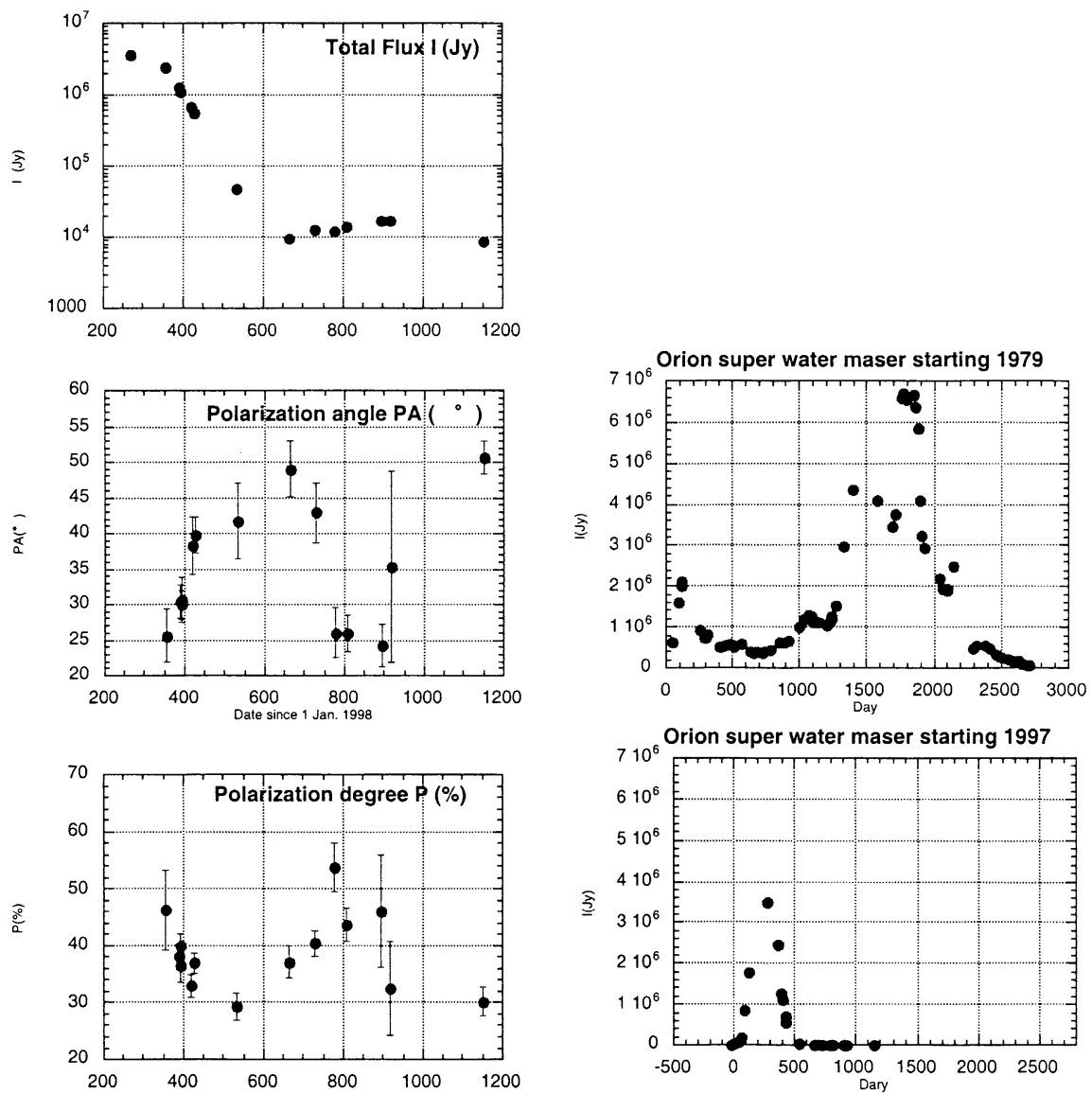

Figure 1. (left)(a) Derived values from our polarization observation as functions of time, (top) total flux density in logarithmic scale, (middle) polarization angle, (bottom) polarization degree. (right)(b) Total flux density variation in time for the previous event adopted from Garay et al. 1989 (top) and present event adopted from Omodaka 1998 and this work (bottom). 
We find that the total flux decreased by almost half within a month from 21 December 1998. The variation in flux seems to be exponential in time. Kagoshima monitoring result (Omodaka et al. 1998) indicates that the total flux increased by almost twice within a month from 4 December 1997, which suggest that the total flux increase is also exponential during the phase of flux increse. Omodaka et al. (1999) has reported that the total flux of this burst increased up to $3.5 \times 10^{6} \mathrm{Jy}$ from 1995 January to 1998 September. Our result has shown that the peak of this outburst event should have been around September 1998, with total flux density probably exceeding 4 million Jy. Comparison of these measurements suggests that the increase and decrease in the total flux density is almost symmetric with respect to the burst peak, except the period around the peak; both follow exponential curves.

The fractional polarization degrees we find are quite high and exhibit a systematic variation in feature over time as shown in Figure 1a(top). On 21 December 1998, the polarization degree was $46 \%$, which decreased gradually to $30 \%$ by 8 June 1999 as the total flux fell two order of magnitude. There is also systematic variation in polarization angle as shown in Figure 1a (bottom). It was $25^{\circ}$ in 1998 December 21 and shifted almost uniformly to $40^{\circ}$ in two months, though varying less rapidly since. Small flare was followed after the minimum of total flux. During the flare the polarization feature seems to have varied in the same manner as the burst.

\section{Discussion}

The present bursting maser spot has an elongated structure with aspect ratio of about 10 and position angle $-40^{\circ}$, as observed by the VLBI Space Observatory Program (VSOP) in March 1998 (e.g. Horiuchi et al. 1999). Assuming that the burst spot has not change it's structure significantly, we find that the polarization angle, ( $E$-vector angle), is nearly perpendicular to the elongation of the bursting spots, similar to the superburst in 80's (Matveyenko et al. 1988).

The high polarization observed in this region suggests the presence of aligned magnetic fields in the maser source, though the maser polarization can be caused also by anisotropic pumping. According to the theory assuming the presence of magnetic field (Deguchi, Watson 1986), the degree of linear polarization increases with degree of saturation, hence with total intensity. For both events during decay the theory fits observation. However for the pre-burst and burst phases, this is not the case. The fact that the time scale of total flux variation is much smaller than that of linear polarization variation may indicate that the bursting event is caused by a purely geometrical effect, a combination of several relatively stable masing spots probably sharing similar polarization characteristics while polarization variation represent some variation of the internal condition in each maser component. Although no computation for solving the radiative transfer equation has been performed for the case of time-dependent interacting maser spots, it is tempting to relate the total intensity variation to some intrinsic linear polarization variation of several maser spots located along the line of sight.

The mechanism of such extremely high brightness masers may be related to the several interacting masers located along the line of sight (Deguchi, Watson 1989, see also Elitzur et al. 1991, Kylafis, Pavlakis 1992). Boboltz et al. (1998) 
has suggested that the symmetric intensity variation of a water maser flare in W49 observed from December 1989 through 1990 can be explained with two interacting maser clouds model; a system with a maser passing across the line of sight toward another background maser. Figure $1 \mathrm{~b}$ for the present burst shows a symmetric variation of the total flux before and after the burst peak, which suggests that the interacting masers model is applicable.

On the other hand, the polarization characteristic seems not to vary symmetry in time around the peak of total intensity. Our result shows that the polarization degree decreased monotonically during the decay phase. To the authors knowledge no polarization monitoring results have been published for the burst phase of the present event. However, a VLBA polarimetry observation at $22 \mathrm{GHz}$ toward Orion-KL made in February 1997, ten months before the present bursting event was recognized, shows that the pre-bursting spot exhibits about $70 \%$ fractional polarization with an extremely modest total flux density of $30 \mathrm{Jy}$ (Horiuchi et al. 1999). The position of this pre-bursting spot coincides with the position of the present burst to within 2 milli-arcseconds. These results indicate that the polarization characteristics has been changing gradually and monotonically while total flux varied rapidly and symetrically. This systematic increase and decrease of the total flux, and secular decrease of the fractional polarization degree may be a key to understanding the interacting maser model working in Orion-KL.

\section{References}

Abraham Z., Vilas Baoas J.W.S. 1994, AA 290, 956

Boboltz D.A., Simonetti J.H., Dennison, B., Diamond P.,Uphoff J.A. 1998, ApJ 509,256

Deguchi S., Watson W.D. 1986, ApJ 302, 750

Deguchi S., Watson W.D. 1989 ApJ 340, L17

Elitzur M. 1991, ApJ 370, 407

Garay G., Moran J.M., Haschick A.D. 1989, ApJ 338, 244

Horiuchi S., Migenes V., Deguchi S. 1999, Abstracts of XXVIth General Assembly of International Union of Radio Science (URSI), 520

Horiuchi, S. and Kameya, O., 2000, PASJ 52, 545-550.

Kylafis N.D., Pavlakis K.G. 1992, ApJ 400, 344

Matveyenko L.I., Graham D.A., Diamond P.J. 1988, SvA(Lett) 14, 468

Omodaka, T., Maeda T., Mochiduki N. 1998, IAUC 6893

Omodaka, T., Maeda T., Miyoshi M., Okudaira A., Nishio M., Miyaji T., Mochiduki N., Morimoto M., Kobayashi H., Sasao T. 1999, PASJ 51, 333 